\title{
Cholesterol efflux capacity-a new biomarker for cardiovascular risk?
}

HDL cholesterol efflux capacity, a measure of reverse cholesterol transport, is inversely associated with the incidence of cardiovascular events. This finding by Rohatgi and colleagues published in The New England Journal of Medicine was also presented at the AHA Scientific Sessions 2014 in Chicago, IL, USA.

Reverse cholesterol transport from peripheral tissues to the liver is thought to be a protective mechanism against the development of atherosclerotic plaques. HDL cholesterol efflux from macrophages is an important step in reverse cholesterol transport, and in animal models is inversely correlated with atherosclerotic lesion size. The development of new strategies to measure cholesterol efflux capacity prompted the investigators to assess whether this parameter is associated with the incidence of adverse cardiovascular events. "HDL is complex and dynamic, and measuring function of HDL may provide a better clue to HDL's actions," explains Anand Rohatgi from the University of Texas Southwestern Medical Center in Dallas, TX, USA.

The investigators enrolled 2,924 individuals (aged 30-65 years) in this multiethnic cohort, who had no history of cardiovascular disease and were participants in the Dallas Heart Study. Participants were assessed for: fasting blood and urine lipid content, including HDL particle concentration and size; detailed cardiovascular health (determined using noninvasive imaging techniques); body fat composition and distribution; and individual cholesterol efflux capacity. Participants were followed up for a median period of 9.4 years.

Increasing cholesterol efflux capacity was inversely associated with the primary end point of atherosclerotic cardiovascular disease when comparing the highest with the lowest quartiles of cholesterol efflux capacity (HR 0.44, 95\% CI 0.27-0.73), and with the secondary end point of total cardiovascular disease (fourth vs first quartiles of cholesterol efflux capacity: HR 0.52, 95\% CI 0.34-0.80). The association between cholesterol efflux capacity and the primary end point remained significant after adjustment for traditional risk factors, HDL cholesterol level, and HDL particle concentration (HR 0.33, 95\% CI 0.190.55). Furthermore, risk prediction for atherosclerotic cardiovascular disease was significantly improved by adding cholesterol efflux capacity to traditional risk factors (C-statistic from 0.827 to 0.841, $P=0.02$ ).

"Several recent clinical and genetic studies have suggested that HDL cholesterol levels are not a reliable therapeutic target for cardiovascular prevention," says Ulf Landmesser from the Charité-Universitätsmedizin Berlin, Germany, who was not involved in the study. According to Landmesser, "the present results strongly support the concept that determination of HDL functional parameters is likely [to be] more important and promising for the development of potential novel therapeutic strategies [than measurement of HDL cholesterol levels]".

The data suggest that cholesterol efflux capacity could be used as a new biomarker to improve discrimination and reclassification indexes in cardiovascular risk assessment. "Our study is the first to link cholesterol efflux at baseline in humans with no cardiovascular disease to incident atherosclerotic events," adds Rohatgi, who clarifies that future work is needed to "validate these findings in other cohorts, and also to determine the factors that regulate cholesterol efflux in humans".

João H. Duarte

Original article Rohatgi, A., et al. HDL cholesterol efflux capacity and incident cardiovascular events. N. Engl. J. Med. doi:10.1056/NEJMoa1409065

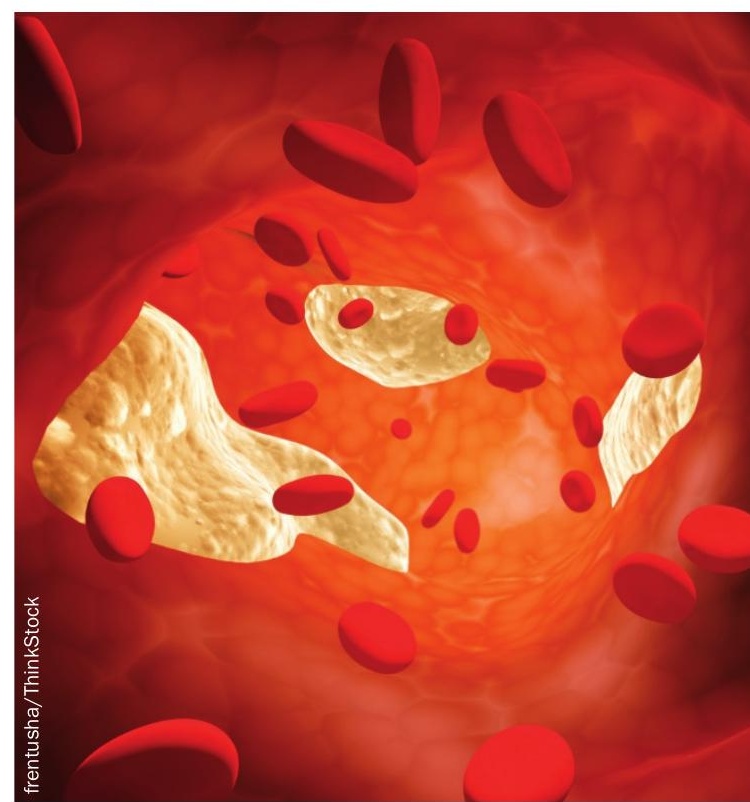

